\title{
BLOCK COMPANION MATRICES, DISCRETE-TIME BLOCK DIAGONAL STABILITY AND POLYNOMIAL MATRICES
}

\author{
HARALD K. WIMMER
}

Abstract. A polynomial matrix $G(z)=I z^{m}-\sum_{i=0}^{m-1} C_{i} z^{i}$ with complex coefficients is called discrete-time stable if its characteristic values (i.e. the zeros of $\operatorname{det} G(z)$ ) are in the unit disc. A corresponding block companion matrix $C$ is used to study discrete-time stability of $G(z)$. The main tool is the construction of block diagonal solutions $P$ of a discrete-time Lyapunov inequality $P-C^{*} P C \geqslant 0$.

Mathematics subject classification (2000): 15A33, 15A24, 15A57, 26C10, 30C15, 39A11.

Keywords and phrases: Polynomial matrices, block companion matrix, zeros of polynomials, root location, discrete-time Lyapunov matrix equation, diagonal stability, systems of linear difference equations.

\section{REFERENCES}

[1] N. Anderson, E. B. SAFF, And R. S. VARGA, On the Eneström-Kakeya theorem and its sharpness, Linear Algebra Appl., 28 (1979), 5-16.

[2] H. Baumgärtel, Analytic Perturbation Theory for Matrices and Operators, Operator Theory: Advances and Applications, Vol. 15, Birkhäuser, Basel, 1985.

[3] A. Ben-Israel and Th. N. E. Greville, Generalized Inverses, 2nd ed., Springer, Berlin, 2003.

[4] St. L. CAMPBEll AND C. D. MEYER, JR., Generalized Inverses of Linear Transformations, Dover Publications, New York, 1991.

[5] I. Gohberg, P. Lancaster And L. Rodman, Matrix Polynomials, Academic Press, New York, 1982.

[6] A. Hurwitz, Über einen Satz des Herrn Kakeya, Tôhoku Math. J., 4 (1913), 89-93; in: Mathematische Werke von A. Hurwitz, 2. Band, 627-631, Birkhäuser, Basel, 1933.

[7] E. Kaszkurewicz And A. Bhaya, Matrix Diagonal Stability in Systems and Computation, Birkhäuser, Boston, 2000.

[8] P. A. REGAlia, On finite precision Lyapunov functions for companion matrices, IEEE Trans. Automat. Contr., 37 (1992), 1640-1644.

[9] H. K. WIMmer, Diagonal matrix solutions of a discrete-time Lyapunov inequality, IEEE Trans. Automat. Control, 43 (1998), 442-445.

[10] H. K. Wimmer, Discrete-time stability of a class of hermitian polynomial matrices with positive semidefinite coefficients, Matrix Methods: Theory, Algorithms and Applications, 409-414, V. Olshevsky and E. Tyrtyshnikov, Editors, to be published by World Scientific.

[11] H. K. Wimmer, Polynomial matrices with hermitian coefficients and a generalization of the Eneström-Kakeya theorem, Oper. Matrices, 2 (2008), 443-454. 